\title{
Construction of confidence intervals for extreme rainfall quantiles
}

\author{
A. T. Silva ${ }^{1}$, M. M. Portela ${ }^{1}$, J. Baez $^{2} \&$ M. Naghettini ${ }^{3}$ \\ ${ }^{I}$ Instituto Superior Técnico, Portugal \\ ${ }^{2}$ Universidad Católica Nuestra Señora de la Asunción, Paraguay \\ ${ }^{3}$ Universidade Federal de Minas Gerais, Brazil
}

\begin{abstract}
In hydrological practice, the design values of extreme rainfalls are generally estimated by means of frequency analysis applied to a finite sample of extreme rainfall values. The most usual approach taken in such analysis is to fit a statistical distribution to an annual maximum series (AMS) built upon one value per year. As an alternative approach, the peaks-over-threshold (POT) technique considers all the peak values above a given threshold and thusly allows for a more rational selection of events to include in the frequency analysis. The research presented in this paper reviews the relative merits of the two aforementioned approaches by analyzing the confidence intervals of extreme rainfall quantiles that result from their application. Despite the usually larger extreme value sample sizes made possible by the POT technique, the estimation of design values by such approach requires the analysis of both the magnitude and the times of arrival of extreme rainfall events. Hence the uncertainty associated with the estimated quantiles results from the combination of the two models applied to ascertain the magnitude and the frequency. Using daily rainfall data samples from Portugal and from Paraguay, four statistical models are applied (two AMS models and two POT models) and a comparison of the estimated quantiles is made. Furthermore, quantile confidence intervals were constructed using both asymptotic theory and the Monte Carlo simulation technique. Such intervals assume an important role in hydrological risk analyses, as they enable the assessment of the uncertainties associated with estimating distribution parameters and quantiles from finite samples of extreme rainfalls. The research was carried out in the scope of the European joint project CapWEM - Capacity development in Water Engineering and Environmental Management
\end{abstract}


- in which eight partner institutions from European and Latin American countries collaborate on improving higher education in the fields of Water and Environmental Management.

Keywords: quantile confidence intervals; peaks-over-threshold; Monte Carlo simulation.

\section{Introduction}

Extreme rainfall events are associated with the risk of infrastructure failure and loss of human lives. Nowadays, studies on the planning, design, and operation of water resources systems often require a comprehensive understanding of the probabilistic behaviour of extreme rainfall events. In general, such behaviour is characterized via frequency analysis applied to the extreme values of rainfall time series, which are treated as random variables. Design rainfall values are usually estimated as quantiles, $x_{F}$, with a predetermined non-exceedance probability, F, of a statistical distribution function that has been fitted to the data and is assumed to adequately describe the probabilistic behaviour of the random variable, $\mathrm{F}_{\mathrm{X}}(\mathrm{x} \mid \boldsymbol{\theta}), \boldsymbol{\theta}=\theta_{1}, \ldots, \theta_{\mathrm{k}}$ being the distribution parameters. Evidently, the point estimator $\hat{\mathrm{x}}_{\mathrm{F}}$ is subject to errors resulting from uncertainties that are intrinsic to the estimation of parameters and quantiles on the basis of finite data samples.

A convenient approach to account for the aforementioned uncertainties consists of constructing $100(1-\alpha) \%$ confidence intervals for the estimated $\hat{X}_{F}$ quantiles, being $(1-\alpha)$ the confidence level, as they summarize the uncertainties associated with estimating distribution parameters and quantiles due to sampling variability, and hence they can indicate the accuracy of the estimates [1]. However, they do not enable the assessment of the uncertainties related to the selection of the probabilistic model, $\mathrm{F}_{\mathrm{X}}(\mathrm{x} \mid \boldsymbol{\theta})$.

The construction of quantile confidence intervals often uses asymptotic theory, which can show that, regardless of the selected model $F_{X}(x \mid \theta)$, the quantiles $\mathrm{x}_{\mathrm{F}}$, are asymptotically Normal with mean $\mathrm{x}_{\mathrm{F}}$ and standard deviation $\mathrm{S}_{\mathrm{F}}$. The latter value corresponds to the standard error of the estimate and can be expressed as a function of the variances and covariances of the estimated parameters and of the partial derivatives of the quantile function. This approach may involve complex analytical procedures, especially when the probabilistic model consists of a three-(or more)-parameter distribution.

As an alternative to the asymptotic theory, one can employ computerintensive simulation procedures, such as the Monte Carlo simulation technique and bootstrap techniques. In broad terms, such techniques involves the estimation of a large number of quantile curves, based on generated data samples with a length equal to that of the original sample.

For a given non-exceedance probability, $\mathrm{F}$, and a fixed confidence level $(1-\alpha)$, the amplitude of the $100(1-\alpha) \%$ confidence interval for the estimate 
$\hat{\mathrm{x}}_{\mathrm{F}}$ decreases as the sample length, $\mathrm{N}$, increases, hence, the obtained estimates are more accurate for larger sample sizes. However, in the hydrological practice, practitioners often have to deal with reduced samples sizes of extreme rainfalls, being that these rainfalls are, generally, scarce. This aspect of extreme rainfall frequency analysis is even more pertinent when it is frequently needed, in design stages, to obtain estimates with return periods that are much larger than the size of the available samples.

In extreme rainfall frequency analysis, the most commonly used sampling technique, known as annual maximum series (AMS), consists of drawing the annual maxima of a $\mathrm{N}$-year rainfall time series with a given duration (more often daily rainfall), resulting in samples with length $\mathrm{N}$ (only one value per year, namely the maximum of that year). Under this approach the amount of data to be selected is limited as it discards extreme rainfall events that despite not being the maxima in the years in which they occur are higher and sometimes much higher than the maxima rainfalls in other years. As an alternative to the AMS sampling technique, the peaks-over-threshold (POT) approach samples all the independent daily rainfall values above a given threshold; hence the resulting samples are not limited to one value per year, thus allowing for a more comprehensive and representative selection of extreme values. The peaks that constitute a POT sample may be modelled by a dual-domain-model that is able to describe both the magnitudes and the times of occurrence of the events. The POT constitutes an attractive alternative to AMS sampling. However, it is analytically more complex and there is some degree of subjectivity involved in its implementation, namely regarding the choice of the threshold.

Despite the formal advantages of the POT technique regarding the higher data availability that it enables, one cannot state $a$ priori that the estimated design values will be more accurate simply because they are supported by more data. In fact, the design extreme rainfall quantiles estimated via POT approach result from a combination of two models (one for the magnitudes and another for the times of arrival) and the associated uncertainties result from both models. Plavšić [2] found that it is not correct to assume that a high number of peaks in a POT sample can 'compensate' for a small number of years of records.

This paper constitutes a brief exploration of some aspects regarding the construction of extreme rainfall quantile confidence intervals. Its main objective is to compare the confidence intervals of extreme rainfall quantiles estimated via the AMS approach to the ones estimated via the POT approach. For that purpose, two long daily rainfall series were used: Carvalho, in Portugal and Villarica, in Paraguay.

The research was carried out in the scope of the European joint project CapWEM - Capacity development in Water Engineering and Environmental Management - Contract no. DCI-ALA/19.09.01/10/21526/ /254922/ALFAIII (2010) 55 -, in the scope of the Alfa III programme, which gathers eight partners, six being from Latin American countries (Argentina, Brazil, Chile, Costa Rica, El Salvador e Paraguay), and the remaining two from European countries (Germany and Portugal). The overall objectives of the project include the capacity building of higher education institutes, primarily in Latin America, for training in water 
resources engineering and environmental management, which encompasses a component on hydrological risk analysis.

\section{Methods}

\subsection{Statistical models}

In this section the formulations of the statistical models used in the study are presented. A prior review of works dedicated to POT frequency analysis (e.g. [3-5]), is recommended for readers who are not familiar with the method, since they thoroughly discuss important and complex aspects of POT sampling which will not be examined in detail in the present paper.

According to Pickands [6], the Generalized Pareto Distribution (GPD) is an appropriate statistical model to model POT data (i.e. the selected peaks over the threshold data, $\mathrm{X}_{\mathrm{OT}}$ ), provided that some operational constraints are adhered to, namely the selection of a proper threshold, the independence of the sampled exceedances, and other complexities involved in the POT sampling technique. The cumulative distribution function of the GPD is given by:

$$
\mathrm{H}(\mathrm{x})=\mathrm{P}\left(\mathrm{X}_{\mathrm{OT}}<\mathrm{x}\right)=1-\left[1-\kappa\left(\frac{\mathrm{x}-\beta}{\alpha}\right)\right]^{1 / \kappa}, \alpha>0, \kappa \neq 0
$$

where $\beta, \alpha$, and $\kappa$ are the location, scale and shape parameters, respectively. The location parameter does not require estimation as it is equal to the defined threshold. Madsen et al. [7] contains a broad discussion on parameter estimation for the GPD, comprehending the maximum likelihood method, the method of moments and the method of probability weighted moments. For $\kappa=0$, the GPD takes the shape of the exponential distribution, ED, as a special case. Formally,

$$
\mathrm{H}(\mathrm{x})=\mathrm{P}\left(\mathrm{X}_{\text {От }}<\mathrm{x}\right)=1-\exp \left(-\frac{\mathrm{x}-\beta}{\alpha}\right), \alpha>0
$$

In this case, the scale parameter, $\alpha$, is estimated by the mean exceedance, regardless of which of the three aforementioned methods for parameter estimation is used.

Usually, the annual number of exceedances is assumed to be Poissondistributed with parameter $\lambda$ (alternatively the Binomial and Negative Binomial distributions can be used). Under this assumption, the distribution function, $\mathrm{F}(\mathrm{x})$, of the annual maxima, $\mathrm{X}_{\mathrm{AM}}$, can be given by $\mathrm{F}(\mathrm{x})=\exp \{-\lambda[1-\mathrm{H}(\mathrm{x})]\}[4,8]$. As a result, if $\mathrm{X}_{\mathrm{OT}}$ follows a GPD (eqn. 1), the distribution of $\mathrm{X}_{\mathrm{AM}}$ is given by:

$$
\mathrm{F}(\mathrm{x})=\mathrm{P}\left(\mathrm{X}_{\mathrm{AM}}<\mathrm{x}\right)=\exp \left\{-\lambda\left[1-\kappa\left(\frac{\mathrm{x}-\beta}{\alpha}\right)\right]^{1 / \kappa}\right\} \kappa \neq 0
$$

where $\beta, \alpha$, and $\kappa$ have the same meaning as before, which is the Generalized Extreme Value distribution (GEV). Eqn. (4) is equivalent to the GEV in its 
classical parameterization, $F(x)=\exp \left\{-[1-\kappa((x-\mu) / \sigma)]^{1 / \kappa}\right\}$, with the same shape parameter, $\kappa$, as the GPD, the scale parameter $\sigma=\alpha / \lambda^{\kappa}$, and the location parameter $\mu=\beta+\sigma[(\lambda \kappa-1) / \kappa]$. Conversely, if $\mathrm{X}_{\mathrm{OT}}$ follows an $\mathrm{ED}$ (eqn. 2), the distribution of $\mathrm{X}_{\mathrm{AM}}$ is given by:

$$
\mathrm{F}(\mathrm{x})=\mathrm{P}\left(\mathrm{X}_{\mathrm{AM}}<\mathrm{x}\right)=\exp \left[-\lambda \exp \left(-\frac{\mathrm{x}-\beta}{\alpha}\right)\right]
$$

which is the Gumbel distribution (or Extreme Value type-1, EV1). Note that eqn. (5) is equivalent to the EV1 in its classical parameterization, $\mathrm{F}(\mathrm{x})=\exp [-\exp ((\mathrm{x}-\mu) / \sigma)]$, with the scale parameter $\sigma=\alpha$, and the location parameter $\mu=\beta+\alpha \ln (\lambda)$.

In rigour, the annual maximum distributions obtained via the POT technique - eqn. (4) and eqn. (5) - are defined only for $x>\beta$, and that for $x=\beta$ they are equal to $\exp (-\lambda)$, which is the probability that no exceedance will happen in a year. It should be noted, however, that the former constraint has little practical significance as, for the usual values of $\lambda$ (not less than 2 or $3[3]$ ), $\exp (-\lambda)$ is very small compared to the relevant quantiles in extreme rainfall frequency analysis ( $\mathrm{F}=0.9$ or higher).

The aforementioned results provide an adequate setup for comparing the POT approach to the AMS approach: if the annual maxima of a series of extreme rainfalls can be modelled, under a POT approach, by a Poisson-GPD model, they can also be modelled, under an AMS approach, by a GEV model. Obviously, an analogous correspondence can be made between the Poisson-ED model and the EV1 model.

\subsection{Construction of quantile confidence intervals}

\subsubsection{Method based on asymptotic theory}

As mentioned in Item 1, asymptotic theory shows that the quantiles with a given non-exceedance probability, $\mathrm{F}$, denoted by $\mathrm{X}_{\mathrm{F}}$, are approximately normaldistributed with mean equal to the estimated quantile $\hat{\mathrm{x}}_{\mathrm{F}}$ and standard deviation $\mathrm{S}_{\mathrm{F}}$. This result enables the construction of $100(1-\alpha) \%$ confidence intervals given by:

$$
\hat{\mathrm{x}}_{\mathrm{F}} \pm\left|\mathrm{z}_{\alpha / 2}\right| \mathrm{s}_{\mathrm{F}}
$$

where $z_{\alpha / 2}$ is the standard normal variate with an $\alpha / 2$ non-exceedance probability. The asymptotic variance, $\mathrm{s}_{\mathrm{F}}^{2}$, for a given k-parameter distribution, $\mathrm{F}\left(\mathrm{x} \mid \theta_{1}, \ldots, \theta_{\mathrm{k}}\right)$, can be calculated using the following equation:

$$
\mathrm{s}_{\mathrm{F}}^{2}=\mathbf{h} \boldsymbol{\Sigma} \mathbf{h}^{\top}
$$


where $\Sigma$ is the variance-covariance matrix of the estimators of the k parameters, $\hat{\theta}_{1}, \ldots, \hat{\theta}_{\mathbf{k}}$ (dependent on the method of estimation), and $\mathbf{h}$ is given by:

$$
\mathbf{h}=\left[\begin{array}{lll}
\frac{\partial \mathrm{q}}{\partial \theta_{1}} & \cdots & \frac{\partial \mathrm{q}}{\partial \theta_{\mathrm{k}}}
\end{array}\right]_{\mathrm{F}}
$$

where $\mathrm{q}$ is the analytical expression of $\mathrm{F}^{-1}(\mathrm{x})$.

Using the Maximum Likelihood (ML) method for estimating parameters, and taking $\gamma=-\ln (\mathrm{F}) / \lambda$, the explicit expression for the asymptotic quantile variance of the Poisson-ED model is given by [7, 9]:

$$
\mathrm{s}_{\mathrm{F}}^{2}=\frac{\alpha^{2}}{\lambda \mathrm{N}}\left[1+\ln (\gamma)^{2}\right]
$$

and of the Poisson-GPD model:

$$
\begin{aligned}
\mathrm{s}_{\mathrm{F}}^{2}= & \frac{\alpha^{2}}{\lambda \mathrm{N}}\left[\gamma^{2 \mathrm{k}}-\frac{1}{\kappa^{2}}(\kappa-1)\left(\gamma^{\kappa}-1\right)^{2}\right. \\
& -\frac{2}{\kappa^{3}}(\kappa-1)\left(\gamma^{\kappa}-1\right)\left(\kappa \gamma^{\kappa} \ln (\gamma)-\gamma^{\kappa}+1\right) \\
& \left.+\frac{1}{\kappa^{4}}(\kappa-1)^{2}\left(\kappa \gamma^{\kappa} \ln (\gamma)-\gamma^{\kappa}+1\right)^{2}\right]
\end{aligned}
$$

The explicit formula for the asymptotic variance of the $\mathrm{F}$ quantile of the Poisson-GPD model given by eqn. (11) was obtained using the asymptotic variance-covariance matrix of the ML estimators of the GPD parameters given by Madsen et al. [7], which takes advantage of the assumption that the sample properties of the exceedance magnitudes are independent of the sample estimates of $\lambda$, hence $\operatorname{Cov}\{\hat{\lambda}, \hat{\alpha}\}=\operatorname{Cov}\{\hat{\lambda}, \hat{\kappa}\}=0$. To the knowledge of the authors, the explicit formula in eqn. (11) has not been given before in the technical literature.

Coles [10] discusses in detail how to determine the asymptotic quantile variances of the EV1 and GEV models, using ML estimation. For the EV1, such determination is not too complicated, given that it is a two-parameter distribution. For the GEV, the procedure is much more complicated and requires numerical evaluation. The R package ismev contains code that performs analyses of the textbook of Coles [10]. This package was used in this research.

It should be stressed that confidence intervals obtained by this method are approximate since they stem from asymptotic results.

\subsubsection{Method based on Monte Carlo simulation}

Compared to the method based on asymptotic theory described above, the Monte Carlo simulation technique is a less arduous, but more computationally expensive, method for constructing quantile confidence intervals. In broad terms, such technique consists of generating a large number of synthetic samples and obtaining from that generated data an empirical distribution of the desired quantiles. 
In the AMS approach, the Monte Carlo simulation algorithm is straightforward:

1. generate a synthetic $\mathrm{N}$-year sample ( $\mathrm{N}$ being the number of years of the original data sample), $\mathbf{x}_{\mathrm{AM}}^{*}$, by randomly sampling from a parent distribution of the annual maxima, $\mathrm{F}(\mathrm{x})$, with parameters estimated from the original sample;

2. apply the ML parameter estimators to the generated sample $\mathbf{x}_{\mathrm{AM}}^{*}$, and, by inversion of the distribution $\mathrm{F}(\mathrm{x})$ with those parameters, estimate the quantile with the desired non-exceedance probability $\mathrm{F}$;

3. repeat the steps 1 and 2 . W times, thus obtaining a vector of $\mathrm{W}$ simulated quantiles;

4. the lower and upper bounds of a $100(1-\alpha) \%$ quantile confidence interval correspond to the $\mathrm{W}(\alpha / 2)^{\text {th }}$ and $\mathrm{W}(1-\alpha / 2)^{\text {th }}$ order statistics of the $\mathrm{W}$ quantiles obtained in step 3 .

Under the POT approach, the simulation algorithm is more complicated because the annual number of exceedances is assumed to be a Poisson distributed random variable and, consequently, the size of the POT sample is variable. The following is a description of the simulation algorithm particularized for GPD distributed exceedances (for ED distributed exceedances, take $\kappa=0)$.

1. For each year $\mathrm{j}$ of the $\mathrm{N}$-year historical sample, generate a random counting variate, $n_{j}$ (the number of over-threshold events in year $\mathrm{j}$ ), from a parent Poisson distribution with parameter $\lambda$ estimated from the original sample, and estimate the 'synthetic' Poisson parameter, $\lambda^{*}$, as $\sum_{\mathrm{j}} \mathrm{n}_{\mathrm{j}} / \mathrm{N}$;

2. generate a random POT sample, $\mathbf{x}_{\mathrm{OT}}^{*}$, of size $\mathrm{N} \lambda^{*}=\sum_{\mathrm{j}} \mathrm{n}_{\mathrm{j}}$, from a parent GPD distribution with parameters equal to those of the original sample;

3. apply the ML estimators to the generated sample $\mathbf{x}_{\mathrm{OT}}^{*}$ to estimate the GPD parameters;

4. using the estimated parameters from step 3. and $\lambda^{*}$ from step 1., invert the annual maxima distribution, $F(x)$, defined by eqn. (5), thereby estimating the quantile with the desired non-exceedance probability $\mathrm{F}$.

5. repeat steps 1 . to 5 . W times, thus obtaining a vector of $\mathrm{W}$ simulated quantiles;

6. the lower and upper bounds of a $100(1-\alpha) \%$ quantile confidence interval correspond to the $\mathrm{W}(\alpha / 2)^{\text {th }}$ and $\mathrm{W}(1-\alpha / 2)^{\text {th }}$ order statistics of the $\mathrm{W}$ quantiles obtained in step 5 .

\section{Data and model fitting}

The data set used in this study consisted of the samples of daily rainfalls in two rain gauges, one located in the North of Portugal (Sample no.1 - Carvalho, N=27 years) and the other in the South of Paraguay (Sample no. 2 - Villarrica, N=48 
years). The main criteria used in data selection for this study was to have one sample from each of the two countries and that the probabilistic behaviour of the extremes of those samples should differ in the sense that one of them could be modelled by the Poisson-ED/EV1 model ensemble, and the other by the PoissonGPD/GEV model ensemble, being that the objective of the paper is to discuss the uncertainty inherent in these two sets of paired models.

As mentioned in 2.1, a proper implementation of POT technique is highly dependent on subjective and complex analyses related POT sampling such as the selection of an adequate threshold, the independence of the sampled exceedances and the validation of the Poisson assumption. A review of Lang et al. [3] is recommended for a thorough discussion on such topics. Such analyses are of paramount importance and have been made in this study; however they are not presented here since the main focus of the paper is the construction of quantile confidence intervals.

The probability plots presented in fig. 1 show that the Poisson-ED/EV1 ensemble is adequate for modelling extreme rainfalls at the Carvalho rain gauge in Portugal (sample no. 1). Under the POT approach, the selected threshold was $30 \mathrm{~mm}$ (sample size of 262).

a)

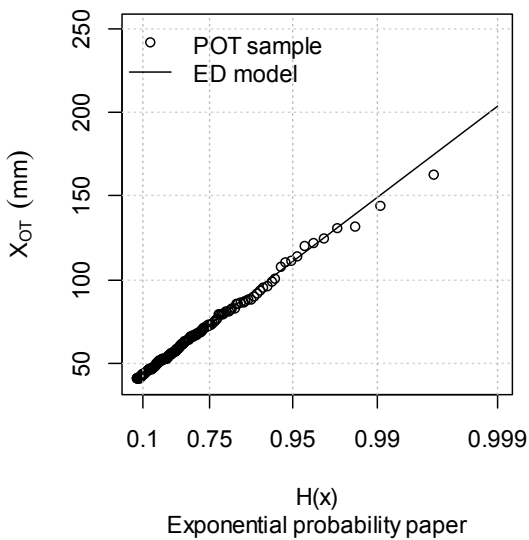

b)

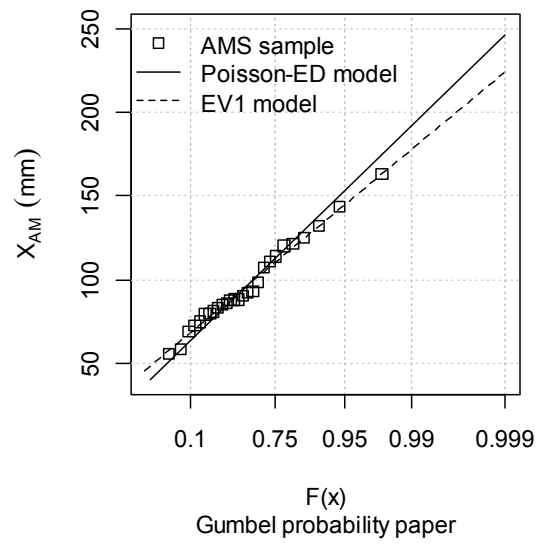

Figure 1: Sample no. 1 - Carvalho (Portugal). (a) Daily rainfalls over a threshold of $30 \mathrm{~mm}$; fitted ED model. (b) Annual maximum daily rainfall; fitted EV1 model (AMS approach); Poisson-ED model (POT approach).

Analogously to fig. 1, fig. 2 shows that the Poisson-GPD/GEV ensemble is adequate for modelling extreme rainfalls at the Villarrica rain gauge in Paraguay (sample no. 2). For this sample, under the POT approach, the selected threshold was $55 \mathrm{~mm}$ (sample size of 291).

It should be noted that the plotting position formula used in figs. 1 and 2 was Gringorten's formula. 
a)

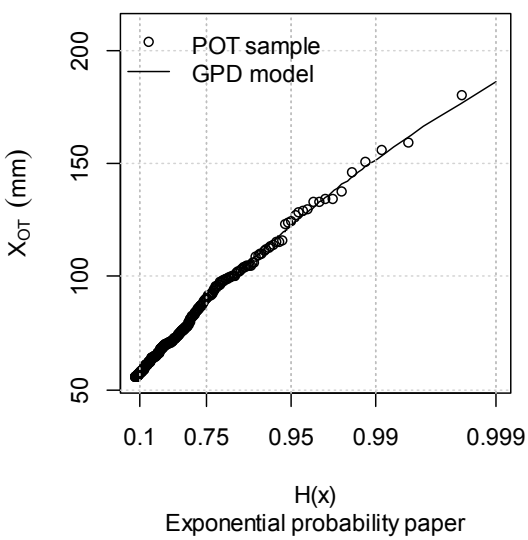

b)

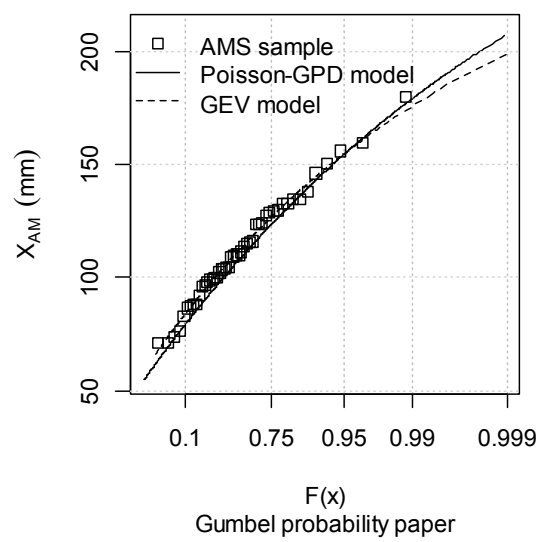

Figure 2: $\quad$ Sample no. 2 - Villarrica (Paraguay). (a) Daily rainfalls over a threshold of $55 \mathrm{~mm}$; fitted ED model. (b) Annual maximum daily rainfall; fitted GEV model (AMS approach); Poisson-GPD model (POT approach).

Figs. $1 \mathrm{~b}$ and $2 \mathrm{~b}$ show that, for both case studies, the distribution of annual maxima obtained via either the POT or the AMS approaches are in fair agreement. Also, since the POT samples are larger and, therefore, more representative of the rainfall extremes at the rain gauges than the AMS samples, the estimates produced via the POT approach are theoretically more supported. All models were fitted using ML estimators [7]. Table 1 includes the respective estimated parameters. That table also summarizes the POT/AMS model ensemble applied to each data sample.

Table 1: Rainfall data. Period of records. Summary of the estimated parameters of the models fitted in figs. 1 and 2 .

\begin{tabular}{|c|c|c|c|}
\hline \multicolumn{2}{|c|}{ Sample no. - Rain gauge (Country) } & 1 - Carvalho (Portugal) & 2 - Villarrica (Paraguay) \\
\hline \multicolumn{2}{|c|}{ Period of records $(\mathrm{N})$} & $1960 / 61-1986 / 87(27)$ & 1956/57 -2003/04 (48) \\
\hline \multicolumn{2}{|r|}{ AMS model } & EV1 & GEV \\
\hline \multirow{3}{*}{ Parameters } & Location, $\mu$ & 84.6489 & 102.4477 \\
\hline & Scale, $\sigma$ & 20.1888 & 21.2592 \\
\hline & Shape, $\kappa$ & - & 0.1315 \\
\hline \multicolumn{2}{|r|}{ POT model } & ED & GPD \\
\hline \multirow{5}{*}{ Parameters } & Poisson rate, $\lambda$ & 9.7037 & 6.0625 \\
\hline & Sample size, $\lambda N$ & 262 & 291 \\
\hline & Location (threshold), $\beta$ & 30 & 55 \\
\hline & Scale, $\alpha$ & 22.9012 & 26.0561 \\
\hline & Shape, $\kappa$ & - & 0.0976 \\
\hline
\end{tabular}




\section{Results}

For sample no 1, and no 2, respectively, figs. 3 and 4 present $95 \%$ quantile confidence intervals for quantiles with a quasi-continuous non-exceedance probability $\mathrm{F}$ (ranging from $\mathrm{F}=0.010$ to $\mathrm{F}=0.999$ ), resulting from the application of both methods introduced in Item 2 (number of synthetic series of $\mathrm{W}=5000$ ).

a)

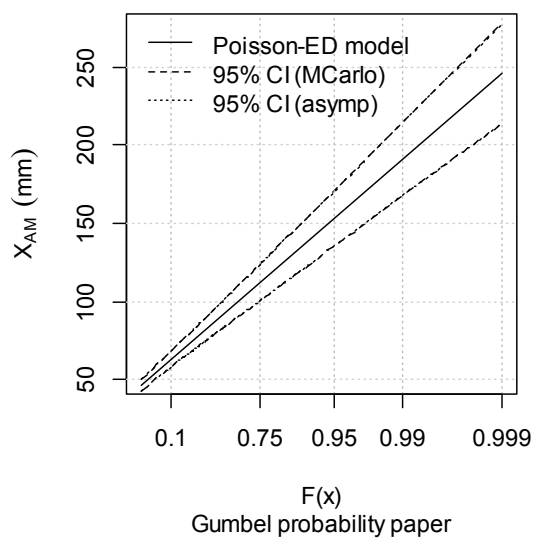

b)

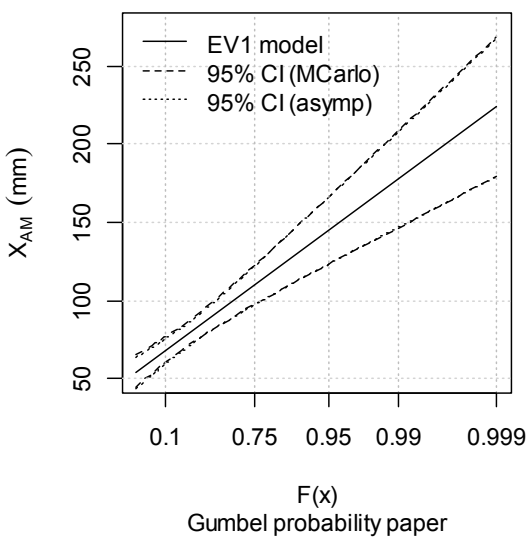

Figure 3: $\quad$ Sample no. 1 - Carvalho (Portugal). Quantile confidence intervals resulting from the application of the asymptotic theory and Monte Carlo simulation methods. (a) POT approach (Poisson-ED model); (b) AMS approach (EV1 model).

The results presented in fig. 3 show that the confidence intervals obtained via asymptotic theory are in almost perfect accordance with the ones obtained via Monte Carlo simulation, under both POT and AMS approaches. This conclusion supports the validity of resorting to computer intensive techniques to find solutions to analytical problems. That figure also clearly shows that there is less uncertainty (more narrow intervals) associated with quantiles estimated by a Poisson-ED model under a POT approach (fig. 3a) than with those estimated by an EV1 model under an AMS approach (fig. 3b), especially for the higher nonexceedance probabilities $(\mathrm{F}>0.9)$, which are the most relevant ones in hydrological design. It is assumed that this is due to the more broad extreme value data selection permitted by POT technique.

The results presented in fig. 4 also show a fair agreement between the confidence intervals obtained via asymptotic theory and with the ones obtained via Monte Carlo simulation, although not as good as in fig. 3. The mismatch between the theoretical and the simulated confidence intervals may suggest that the introduction of a non-zero shape parameter, $\kappa$, in the GPD seems to result in a slight positive skewness in the distribution of higher-order quantiles. This is visible under the POT approach (fig. 4a) but is much more evident under the 
AMS approach (fig. 4b). A significant skewness in quantile confidence intervals suggests that the asymptotic theory may not give a good approximation of the true distribution of those quantiles and, in this case, simulation techniques are preferred. In fig. 4, the formal advantage that supports the application of POT analysis (narrower confidence intervals) is also present but is not so evident as in the Poisson-ED/EV1 ensemble based on sample no. 1.

a)

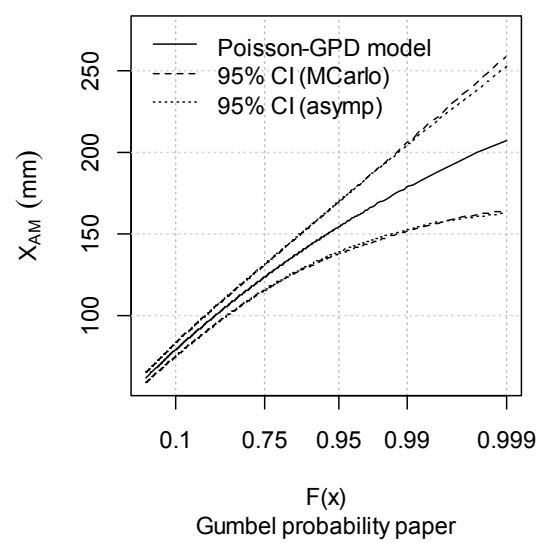

b)

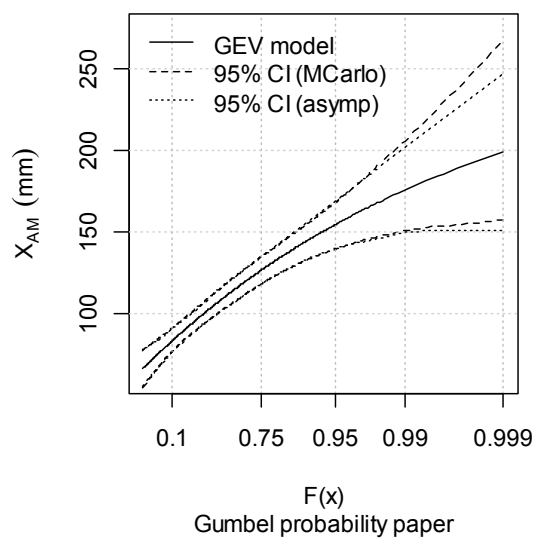

Figure 4: $\quad$ Sample no. 2 - Villarrica (Paraguay). Quantile confidence intervals resulting from the application of the asymptotic theory and Monte Carlo simulation methods. (a) POT approach (Poisson-GPD model); (b) AMS approach (GEV model).

\section{Conclusion}

In frequency analysis of extreme rainfalls, the peaks-over-threshold (POT) technique constitutes an attractive alternative to the more conventional annual maximum series (AMS) technique since it allows for a broader selection of extreme events to include in the samples.

The research presented in this paper discussed the relative merits of the POT and AMS approaches regarding the analysis of uncertainty of the estimated quantiles via the construction of quantile confidence intervals.

Two POT/AMS model ensembles were applied to a data set consisting of two daily rainfall samples (one from Portugal and one from Paraguay) and their quantiles estimated. Subsequently, quantile confidence intervals were constructed using both analytical methods based on asymptotic theory and computational methods based on Monte Carlo simulation algorithms. Such confidence intervals summarize the uncertainties associated with the estimated parameters due to sampling variability.

It was found that it is possible to reduce the uncertainty (obtain narrower confidence intervals) of extreme rainfall quantiles if they are estimated using a 
POT approach, as an alternative to the more commonly used AMS approach. This is due to the larger data availability made possible by POT sampling.

\section{References}

[1] Rao, A.R. and Hamed, K.H., Flood Frequency Analysis, CRC Press, Boca Raton (FL), 2000.

[2] Plavšić, J., Uncertainty in flood estimation by partial duration series, Proceedings of the AMHY-FRIEND International Workshop on Hydrological Extremes, Cosenza, Italy, 2006.

[3] Lang, M., Ouarda, T.B.M.J. and Bobée, B., Towards operational guidelines for over-threshold modeling. Journal of Hydrology, 225, pp. 103-117, 1999.

[4] Naghettini, M. and Pinto, E.J.A., Hidrologia Estatística, CPRM, Belo Horizonte, 2007.

[5] NERC, Flood Studies Report I - Hydrological studies, National Environment Research Council, London, 1975.

[6] Pickands, J. Statistical inference using extreme order statistics. Annals of Statistics, 3(1), pp. 119-131, 1975.

[7] Madsen, H., Rasmussen, P.F. and Rosbjerg, D., Comparison of annual maximum series and partial duration series methods for extreme hydrologic events 1. At-site modelling. Water Resources Research, 33(4), pp. 747-757, 1997.

[8] Davison, A.C. and Smith, R.L., Models for exceedances over high thresholds. Journal of the Royal Statistical Society B, 53(3), pp. 393-442, 1990.

[9] Cunnane, C. A particular comparison of annual maxima and partial duration series methods of flood frequency prediction. Jounal of Hydrology, 18, pp. 257-271, 1973.

[10] Coles, S. An Introduction to Statistical Modeling of Extreme Values, Springer, London, 2001. 